

\title{
Real-Time Water Demand Forecasting System through an Agent-Based Architecture
}

\author{
Borja Ponte $^{1, *}$, David de la Fuente ${ }^{1}$, Raúl Pino ${ }^{1}$, and Rafael Rosillo ${ }^{2}$ \\ ${ }^{1}$ Polytechnic School of Engineering, University of Oviedo, Campus de Viesques s/n, 33204, Gijón (Spain) \\ \{ponteborja,david,pino\}@uniovi.es \\ *Corresponding author \\ ${ }^{2}$ Faculty of Economics and Business, University of León, Campus de Vegazana s/n, 24071, León (Spain) \\ rrosc@unileon.es
}

\begin{abstract}
Water policies have evolved enormously since the Rio Earth Summit (1992). These changes have led to the strategic importance of Water Demand Management. The aim is to provide water where and when it is required using the fewest resources. A key variable in this process is the demand forecasting. It is not sufficient to have long term forecasts, as the current context requires the continuous availability of reliable hourly predictions. This paper incorporates artificial intelligence to the subject, through an agent-based system, whose basis are complex forecasting methods (BoxJenkins, Holt-Winters, Multi-Layer Perceptron Networks and Radial Basis Function Networks). The prediction system also includes data mining, oriented to the pre and post processing of data and to the knowledge discovery, and other agents. Thereby, the system is capable of choosing at every moment the most appropriate forecast, reaching very low errors. It significantly improves the results of the different methods separately.
\end{abstract}

Keywords: agent-based architecture; Artificial Neural Networks, Box-Jenkins; data mining; demand forecasting; Holt-Winters; hourly forecasting; Multi-Layer Perceptron; Radial Basis Functions; Water Demand Management.

\section{Biographical notes:}

Borja Ponte is a PhD student and lecturer at the Department of Business Administration of the University of Oviedo. His Master's Thesis "The Bullwhip Effect in Supply Chains: An Approach based 
on Artificial Intelligence Techniques", qualified with honors, represents its introduction into the world of research, trying to combine the fields of Supply Chain and Multiagent Systems. He has already published papers in several journals and presented papers at several international conferences.

David de la Fuente, Full Professor, Operations Management \& Management Science. PhD Management Science (1987, Universidad de Oviedo) (Extr. Thesis Award). Vice Dean - Industrial Engineering School, University of Oviedo, Spain, (1987-1991). Invited professor at several Latin-American Universities (in Bolivia, Argentina, Cuba, El Salvador etc.) Member of the International Scientific Committee of 30 Technical Conferences. Director of several research projects (European, national and regional), and contracts with companies. 20 Papers in international journals indexed by JCR and SJR. Member of POMS, EurOMA and INFORMS, etc.

Raúl Pino graduated in Industrial Engineering in 1992 at University of Oviedo (Spain), and gained his Ph. D. from the University of Oviedo in 2000. He is currently Senior Lecturer at the Gijón Polytechnic School of Engineering. His current research interests are in the areas of artificial intelligence, forecasting, simulation and manufacturing. He has published a great number of research papers in a number of leading journals.

Rafael Rosillo is a Senior Lecturer in Business Management at University of León. PhD in Business Management, he has also a MBA and a degree in Computer Engineering. His researches are focused on Artificial Intelligence, Stock Markets, Forecasting and Supply Chain. He has collaborated like visiting researcher in John Moores University (Liverpool) and University College of Dublin. He is author of several publications in different national and international journals and chapters of books. He has participated in several international scientific conferences and several research projects.

\section{Introduction}

Water is a basic resource for human life and for the economic growth of any region. The traditional water management is based on extracting new water resources and making them serve human purposes. This way, large amounts of public money have been invested to finance water projects in order to stimulate the economic development. However, this approach, which is based on the supply increase, has barely taken into account that water is a finite and fragile resource, whose availability depends on the functioning of the hydrological 
cycle. For this reason, the concept of Water Demand Management has significantly evolved over the last years. Especially since the Earth Summit held in Rio de Janeiro (1992), due to the pressures generated by the population growth, the urbanization and the industrialization, the strategic importance of WDM is understood, as well as its relevance in the efficiency of municipal management (Mohamed and Savenije, 2000). Brooks (2006) proposes an operational definition of WDM with five components: (1) reducing the quantity or quality of water required to accomplish a specific task; (2) adjusting the nature of the task so it can be accomplished with less water or lower quality water; (3) reducing losses in movement from source through use to disposal; (4) shifting time of use to off-peak periods; and (5) increasing the ability of the system to operate during droughts.

A key aspect in any water management plan is demand forecasting. An accurate forecast can minimize the water used to meet demand, but besides it also results in a reduction of the energy used in the process of catchment, purification and distribution of water and it also produces a saving in the resources spent on sizing the storage and distribution system. The traditional approach to water management required only long term forecasts expressed in annual demands or even decades (Willsie and Pratt, 1974). They were enough for the design of the system (capacity of the tanks, dimension of the pipes and connections between the various nodes) and for the development of plans for meeting the demand. Nevertheless, with the passing of time, this horizon has become shorter. In fact, for attaining high efficiency in the WDM, reliable short-term forecasts are required (Gato et al., 2007). Daily forecasts involve the implementation of supply plans, by setting the system to that effect. The next step is hourly water forecasting. According to Herrera et al. (2010), the ready availability of hourly predictions of water demand is crucial due to three main reasons: it allows to determine the optimal regulation and pumping systems to meet the predicted demand, which promotes energy efficiency (operative point of view); it allows to combine water sources in the most appropriate way to achieve a preset standard in the supply water (quality point of view); and it allows to detect failures and network losses through the comparison of the actual and expected flow (vulnerability point of view).

The literature on the subject contains several works of short term demand forecasting. The first one was written by Maidment et al. (1985), who used statistical models (in particular, ARIMA methodology) to express the daily water demand as a function of ambient temperature and volume of rain. In a later work, the same authors (Maidment and Miaou, 1986) 
proved its efficiency for nine American cities. Other authors (e.g., Shvarster et al., 1993; An et al., 1995) followed this line, using statistical methods and climatic factors in the prediction. Lertpalangsunti et al. (1999) were pioneers in the introduction of artificial intelligence (AI) in the study. They developed a complex forecasting system, which integrated fuzzy logic, artificial neural networks (ANNs) and case-based reasoning, which was tested with high efficiency to forecast the daily water demand in the city of Regina (Canada). Msiza et al. (2007) introduced support vector machines (SVM) in the subject, in order to compare its performance with ANNs, using two different structures: Multi - Layer Perceptron (MLP) and Radial Basis Function (RBF). They conducted the study on the daily demand of the province of Gauteng (South Africa) and the ANNs outperformed the SVM. Herrera et al. (2010) further reduced the time horizon and they evaluated six predictive models (ANNs, projection pursuit regression, multivariate adaptive regression splines, SVM, random forests and a weighted pattern-based models) in forecasting the hourly demand of the city of Valencia (Spain). The authors justify that in this modern environment the ready availability of hourly water demand predictions is crucial. Bio-inspired algorithms have also been used in other aspects around WDM - e.g. Liu and Lv (2009) used the particle swarm optimization algorithm to forecast the residual life of underground pipelines.

On the one hand, one of the main conclusions of the literature review is that these advanced methodologies are proven to give a great performance in the forecasting of short term water demand, both daily and hourly. There are not big differences between their results, as the choice of the optimal one depends on the characteristics of the study period and its recent past. On the other hand, most of the authors use climatic factors in the predictions, as they lead to improve the results. However, the just-in-time availability of these climatic factors in order to perform the hourly forecasting could be a hurdle difficult to overcome by a realtime WDM system. Therefore, considering those factors could be a constraint for the implementation. This way, Nasseri et al. (2011) developed a model based on AI techniques (genetic algorithms and Kalman filter) with excellent results, taking only in consideration data from previous demand.

Under these circumstances, this paper shows the development of a system for the real-time water demand forecasting based on AI techniques. More specifically, we use an agent-based architecture to construct the system, whose core are the advanced forecasting agents but it is also formed by other agents which carry out other important functions, which will be de- 
scribed next. The system continuously receives values from water hourly demand and it is capable of choosing the most reliable forecasting technique at each moment. This way, it could be implemented in different scenarios, as it has the ability of adapting to them. So, after the literature analysis, the idea of this article is to combine different tools in order to obtain a forecasting system with greater accuracy, even without the availability of real-time information about the climatic factors. The great advantage of using the agent-based architecture is that this forecasting system can be integrated into a larger management system built under the same principles.

Our investigation line has been the following: (1) Problem world and problem statement; (2) Development of the conceptual model; (3) Implementation of the forecasting methods; (4) Construction of the real-time water demand forecasting system; (5) Experimentation and obtaining results; and (6) Problem analysis and deriving conclusions. Such work structure is spread across this paper, which is divided into four main sections, including this introduction. Section 2 describes the forecasting system that we have created, with the different agents that form it and their purpose, the structure that encompasses all and the relationships between them. Section 3 contains the numerical results after testing the system with hourly water demand time series and the discussion thereof. Finally, section 4 presents the main conclusions that we have obtained based on the stated objectives, as well as the future investigation lines.

\section{Description of the Real-Time Water Demand Forecasting System}

Figure 1 shows schematically the forecasting system that we have devised and implemented. It consists of nine different agents: the Interface Agent, the Storage Agent, the Data Mining Agent, the Fitness Agent and the five Forecasting Agents (Naïve Agent, Box-Jenkins Agent, Holt-Winters Agent, MLP-NN Agent and RBF-NN Agent). It should be noted that we are using forecasting techniques of different nature. The system receives hourly data about the water demand from the measurement equipments and shows the real-time forecast to the user, in order to the decision-making process. The agent-based approach also allows its connection to a larger management system. Below, we detail the functionality of each agent, and the relationships among them. 


\subsection{Transmission Agents}

The Interface Agent connects the forecasting system with its environment. That is to say, it acts as the intermediary between the rest of the agents and the outside with the aim of reaching the homogeneity in the agent-based system. Thus, it works in a double way: (1) it transmits the demands received hourly from the measurement equipment to the data base; and (2) it transfer the best forecast at each hour to the outside.

The Storage Agent manages a database attached to the system that saves hourly the values of both actual demands and the forecasts performed by the five agents. Besides, it also saves the best forecast performed at every hour. It is necessary to store all this information (not only the best forecast) because past forecasts will influence in the selection of the best forecast in future. Therefore, the Storage Agent is in permanent contact with the Interface Agent and the Data Mining Agent to store and move information from the outside to the other forecasting agents (demand flow), and in the opposite direction (forecasting flow).

The Data Mining Agent carries out the pre processing of the information stored in the database and the post processing of the predictions. On the one hand, this involves extracting the last 1020 hourly demands ( 6 weeks +12 hours, see section 2.9 ) from the database. It has proven to be a suitable time period, in terms of identifying the seasonality and trend of the series. On the other hand, with the aim of performing the neural networks forecasting, it involves the creation of thirteen time series with the demands displaced (displacement from 1 to 4 , from 24 to 28 and from 168 to 172 hours, given the double periodicity of the series, and because the other values have not proved to be significant) to find inference rules and try to explain each demand based on past data. In addition, the Data Mining Agent is connected with the Forecasting Agent, to perform transformations on the variables (e.g., logarithmic, differentiation or quantification of non numerical variables) when it is needed. 


\subsection{Forecasting Agents}

These agents are the real core of the real-time water demand forecasting system. We are using naïve models, classical statistical methods and AI-based techniques, in order to try to combine the advantages of each alternative.

The Naïve Agent performs the demand forecast using a naive method, which estimates the hourly demand $\left(\widehat{D}_{t}\right)$ as the demand in the previous hour $\left(\mathrm{D}_{t-1}\right)$, adjusted by the increase (or decrease) in the demand in the same time interval of the previous week $\left(D_{t-168}-D_{t-169}\right)$, by (1). This is a very simplified model -and hence it requires a insignificant calculation time- but it offers good performance in regular series, like the one we have.

$$
\widehat{D}_{t}=y_{t}=D_{t-1}+\left(D_{t-168}-D_{t-169}\right)
$$

The Box-Jenkins Agent performs the forecast using the ARIMA methodology (Box and Jenkins, 1970). These models can be expressed by $(p, d, q)(P, D, Q)_{n}$, where the parameters are the orders of autoregression (p, P), differentiation (d, D) and moving average (q, Q). Lowercase variables are not seasonal components, while the uppercase ones are seasonal, with periodicity $n$. In our case, $n=168$. These models consider that the future value of the differentiated variable $\left(\widehat{\Delta}^{\mathrm{d}} \mathrm{D}_{\mathrm{t}}\right)$ can be expressed as a function of past observations $\left(\mathrm{D}_{\mathrm{t}-\mathrm{i}}, \mathrm{i} \in\right.$ $[1, \mathrm{n}])$ and a random error $\left(\varepsilon_{\mathrm{t}-\mathrm{j}}, \mathrm{i} \in[1, \mathrm{q}]\right)$. It is expressed in (2), where $\Delta$ is the differentiation operator, $\gamma$ is the constant model, $\varphi_{\mathrm{i}}$ are the parameters associated with autoregression, and $\theta_{\mathrm{j}}$ are the parameters associated with the moving average.

$$
\begin{aligned}
\widehat{\Delta^{d} D_{t}=y_{t}=} & \gamma+\sum_{i=1}^{p} \varphi_{i} \Delta^{d} D_{t-i}+\sum_{k=1}^{P} \varphi_{k n+p} \Delta^{d} D_{t-(k n+p)}-\sum_{j=1}^{q} \theta_{j} \varepsilon_{t-j} \\
& -\sum_{m=1}^{Q} \theta_{m n+q} \varepsilon_{t-(m n+q)}
\end{aligned}
$$

The method of obtaining the statistical model $(p, d, q)(P, D, Q)_{n}$ associated with each time series is based on the sequential process of: (1) identifying the possible model; (2) parameter estimation; and (3) validation. It is repeated until the model is validated through their autocorrelation functions and until its forecasts are validated by a given error criterion. In our case, the Box-Jenkins Agent seeks the model that best fits the input time series, using the 
following statistics for the comparison of the different proposed models: goodness-of -fit according to the criteria of MAPE; residual simple autocorrelation function (ACF); and residual partial autocorrelation function (PACF). The method of obtaining the model and calculating the coefficients is described in more detail in Box and Jenkins (1970).

The Holt-Winters Agent uses the Holt-Winters exponential smoothing method to forecast. Its base is a simple exponential smoothing, which express the demand as a weighted average between the demand and the forecast of the previous period. Holt (1957) modified this model so that it can be applied in trended series and Winters (1960) adapted it for series with seasonality. There are two main Holt-Winters models, depending of the type of seasonality: (1) Multiplicative; and (2) Additive. These models can be mathematically expressed by (3) and (4), in the previous order, where $y_{t}$ represents the forecast, $\overline{\mathrm{R}_{\mathrm{t}-1}}$ is the estimate of the deseasonalized level or overall smoothing in the previous period, $\overline{\mathrm{G}_{\mathrm{t}-1}}$ is the estimate of the trend or smoothing of the trend factor in the previous period, and $\overline{\mathrm{S}_{\mathrm{t}-\mathrm{L}}}$ is the estimate of the seasonal component or smoothing of $\mathrm{L}$ (the seasonal index) periods ago. In our case, $\mathrm{L}=168$.

$$
\begin{aligned}
& \widehat{D}_{t}=y_{t}=\left(\overline{R_{t-1}}+\overline{G_{t-1}}\right) \cdot \overline{S_{t-L}} \\
& \widehat{D}_{t}=y_{t}=\overline{R_{t-1}}+\overline{G_{t-1}}+\overline{S_{t-L}}
\end{aligned}
$$

It should be noted that each one of the previous parameters depends on a different smoothing constant. The procedure for the estimates of model parameters is detailed, among others, in Kalekar (2004). In our case, the Holt-Winters Agent looks for the model that best fits the input time series using the same statistics for the comparison of three alternatives (the multiplicative model, the additive model, and the simple seasonal model, where there is no trend) as the one used in the Box-Jenkins Agent.

The $M L P-N N$ Agent and the $R B F-N N$ Agent estimate the hourly demand through an Artificial Neural Network (ANN) with three levels: an input layer (predictor variables, which are obtained by means of the Data Mining Agent), a hidden layer (composed by nodes that, during optimization process, attempt to functionally map the model inputs to the model outputs) and an output neuron (variable to predict). Figure 2 shows schematically the general structure of the ANN that we have used. 


\section{PLEASE INSERT FIGURE 2 HERE}

In both cases, the data available for each forecast (1008 hourly water demands) are randomly separated into two groups. $70 \%$ is oriented to the batch training of the network, by means of the back-propagation algorithm. The remaining 30\% has been directed for verifying the network. We use different stopping criteria (maximum number of steps without reducing error: 1000; maximum workout time: 1 minute; minimal relative change in training error: 0.0001; minimal relative change in error rate training: 0.001). The steps for developing the ANNs are similar to those detailed in Pino et al. (2008).

There are various ANN architectures. On the one hand, the MLP-NN Agent focus on the Multi-Layer Perceptron (MLP). These are networks that have more than one layer of adaptive weights. A MLP has three layers of units taking values in the range $0-1$, and each layers is nourished with the previous ones. Any number of weighted connections can be used, but MLPs with two weighted connections are very much capable of approximation just about any functional mapping (Bishop, 1995). The MLP can be mathematically represented by (5), where $y_{t}$ represents the output (forecast), $f_{\text {outer }}$ represents de output layer, $f_{\text {inner }}$ represents the input layer transfer function, $w_{x y}$ represents the weights and biases $(i \in[1,17]$ refers to the input neurons and $\mathrm{j} \in[1, \mathrm{n}]$ refers to the hidden neurons) and ${ }^{(\mathrm{z})}$ represents the $\mathrm{z}$-th layer.

$$
\widehat{D}_{t}=y_{t}=f_{\text {outer }}\left[\sum_{j=1}^{n} w_{1 j}^{(2)} \cdot f_{\text {inner }}\left(\sum_{i=1}^{17} w_{j i}^{(1)} \cdot x_{i}+w_{j 0}^{(1)}\right)+w_{10}{ }^{(2)}\right]
$$

On the other hand, the RBF-Agent performs the forecast according to the Radial Basis Function (RBF) Architecture. In the RBF, the activation of the hidden unit is determined by the distance between the input vector and the prototype vector, leading to a two stage procedure (Bishop, 1995): (1) Determination of the centre of the network using unsupervised methods; and (2) Determination of the final-layer weights. Hence, the RBF networks provide an interpolation function -called basis functions-, which passes through each and every data point. It can be mathematically represented by (6), where $y_{t}$ represents the output (forecast), $w_{x y}$ 
represents the weights and biases $\left(\mathrm{j} \in[1, \mathrm{n}]\right.$ refers to the hidden neurons) and $\varphi_{\mathrm{j}}$ represents the activation function of the output layer.

$$
\widehat{D}_{t}=y_{t}=\sum_{j=1}^{n} w_{1 j} \cdot \varphi_{j}
$$

\subsection{Fitness Agent}

The Fitness Agent selects the best forecast at each moment through the comparison of the last demands and the forecasts performed by the five Forecasting Agents. It uses the criterion of the minimum MAPE (mean absolute percentage error), introduced by Makridakis (1993). After evaluating different options, we have obtained the best results when the MAPE is calculated for the last 12 hours, so this agent uses this number for the selection. Figure 3 synthesises the time horizon of the forecasting process, and the role of the Fitness Agent within the whole system.

PLEASE INSERT FIGURE 3 HERE

\section{Numerical Application and Discussion of the Results}

In order to test the forecasting system, we have used a simulated time series with over 15,000 data points, which represents the hourly water demand in the city of Gijón (a municipality of 300,000 inhabitants in the north of Spain) during 21 months (years 2009 and 2010). To obtain it, we have based on the monthly demand of the city, a distribution model of hourly water demand for a city in south-eastern Spain (Herrera et al., 2010), and random parameters. It should be noted that in this city, $71 \%$ of invoiced water is oriented to domestic use, $23 \%$ of this water has an industrial use, and the remaining $6 \%$ is managed by the city council.

The time series of the hourly water demand is a complex series with a double seasonality. On the one hand, it has a daily periodicity, namely every 24 hours the series has a similar 
structure. There is a sharp decrease from $19 \mathrm{~h}$ until $02 \mathrm{~h}$, when demand stabilizes around a daily minimum, until 06h. Then, it grows until 11am, where it sets a first local maximum. From there, demand undergoes a slight decline to local minimum at $14 \mathrm{~h}$, at which time it surges to a second local maximum at $19 \mathrm{~h}$. The mentioned times are approximate and vary according to the season of the year. On the other hand, there is a weekly periodicity (168 hours), as the structure is repeated every week, with a significantly lower consumption on Saturdays and even more on Sundays. Moreover, the time series does not remain in a constant range, but it exhibits different trends in both mean and variance, throughout the year. To illustrate the explanation, figure 4 represents two parts of the time series.

\section{PLEASE INSERT FIGURE 4 HERE}

Data of the hourly water demand time series can be divided into three groups: (1) working days; (2) weekend; and (3) holidays (and days around them, whose forecast could be crucially affected by holidays). After several tests, Table 1 presents the numerical results for two standard cases of each group. In every test, we stand out the MAPE of the forecast performed by the system in the last column (Forecasting MAPE), which is chosen between the various methods and corresponds to the Forecasting Agent with minimizes the MAPE (Fitness MAPE). This Forecasting Agent is stood out in the second column.

\section{PLEASE INSERT TABLE 1 HERE}

In the forecasting of working days, all methods achieve low forecast errors (between 1.12\% and $2.56 \%$ for the two tests shown). Therefore, all of them are capable of understanding the running of the series quite accurately. Even the Naïve Agent, which adopts an oversimplification, provides good results given the regular nature of the series. The statistical models of Box-Jenkins (ARIMA) and Holt-Winters (exponential smoothing) generally improve the results. Nevertheless, as expected, the introduction of artificial intelligence in the model, through ANNs, causes a greater decrease in the MAPE. The results of the RBF and MLP 
structures have a similar goodness-of-fit -there is no significant difference in its performance. By way of example, Figure 5, which represents the forecasting time period for test I, shows what we have explained.

\section{PLEASE INSERT FIGURE 5 HERE}

On weekend forecastings, all methods increase substantially the error generated. This is easily understandable, since the influence of the working days on the model is much higher. Statistical models in this case are less robust, as they show high variability in the goodness of their results. In some tests, they achieve low forecast errors but in others they are not able to accurately grasp the series. The RBF structure in ANNs shows a similar effectiveness. However, the $M L P-N N$ Agent offers the best performance, reaching a MAPE less than 3\% in all cases analyzed. By way of illustration, Figure 6 shows the demand and the forecasts performed by the various agents in test III.

The problems of statistical models are more evident on holidays and days around them. On the one hand, the system is not capable of adapting its structure in atypical days, while ANNs can manage it (see test $V$ ). On the other, the presence of a holiday in the days before the forecasting period introduces a distortion in the series model that deviates slightly the forecast (see test VI). Therefore, in this last group, the differences between the different methodologies are amplified and AI allows improving strongly the forecast. This can be shown in Figure 7, which displays the demand and the forecasts performed by the various agents in test VI. In holidays, again, the forecasting of ANN with MLP structure is more robust than the ANN with RBF structure. 


\section{Conclusions and Next Steps}

This paper presents an application of agent-based architecture in hourly demand forecasting, a key aspect in Water Demand Management (WDM). The cores of the system are advanced statistical models (ARIMA and Holt-Winters exponential smoothing) and artificial intelligence (AI) techniques, such as Multi-Layer Perceptron (MLP) and Radial Basis Functions (RBF) Artificial Neural Networks (ANNs). Tests that have been carried out demonstrate the effectiveness of the real-time forecasting system, which selects at each moment the best forecast. Obviously, there is no way to ensure that the system always selects the prediction that will generate the lower error in future, but tests show that if the forecasting method selected is not optimal, it is closer to the optimum. The goodness-of-fit of each technique depends on the characteristics of the forecasting period, although MLP is the most robust method.

The multi-agent environment draws a very appropriate approach to tackle the problem, as the system provides at all times the forecast which it understands as the best. Under these circumstances, it allows the addition of new intelligent forecasting tools by means of new Forecasting Agents, without varying the rest of the system. In addition, this approach has enormous potential in increasing its functionality, because it allows to complete the study by adding new agents. This way, this real-time water demand forecasting system will be integrated in a larger system aimed at optimizing the management.

\section{Acknowledgments}

This research work has been supported by the Government of the Principality of Asturias, through the Severo Ochoa program (reference BP13011). We would also like to acknowledge the help provided by the municipal water company of Gijón in providing data Finally, we would like to thank the Banco Sabadell Foundation for their financial support. 


\section{References}

An, A., Shan, C. C., Cercone, N. and Ziarko, W. (1995) 'Discovering rules from data for water demand prediction', Proceedings in the Workshop on Machine Learning and Expert System, pp. 187-202.

Bishop, C. M. (1995) 'Neural Networks for Pattern Recognition', Oxford University Press.

Box, G. E. P. and Jenkins, G. M. (1970) 'Time Series Analysis: Forecasting and Control', Holden Day, San Francisco.

Brooks, D. B. (2006) 'An Operational Definition of Water Demand Management', International Journal of Water Resources Development, Vol. 22(4), pp. 521-528.

Gato, S., Jayasuriya, N. and Roberts, P. (2007) 'Temperature and rainfall thresholds for base use urban water demand modeling', Journal of Hydrology. Vol. 337, pp. 364-376.

Herrera, M., Torgo, L., Izquierdo, J. and Pérez-García, R. (2010) 'Predictive models for forecasting hourly urban water demand', Journal of Hidrology, Vol. 387, pp. 101-140.

Holt, C. C. (1957) 'Forecasting seasonals and trends by exponentially weighted moving averages' ONR Memorandum, Vol. 52, Pittsburgh, PA, Carnegie Institute of Technology.

Kalekar, P. S. (2004) 'Time series Forecasting using Holt-Winters Exponential Smoothing', Kanwal Rekhi School of Information Technology, Tech. Rep.

Lertpalangsunti, N., Chan, C., Mason, R. and Tontiwachwuthikul, P. (1999) 'A tool set for construction of hybrid intelligent forecasting systems: application for water demand prediction', Artificial Intelligence in Engineering, Vol. 13, pp. 21-42.

Liu, Q. M., and Lv, W. (2009) 'The forecasting residual life of underground pipeline based on particle swarm optimisation algorithm', International Journal of Bio-Inspired Computation, Vol. 1 (4), pp. 270-275.

Maidment, D. and Miaou, S. (1986) 'Daily water use in nine cities', Water Resources Research, Vol. 22 (6), pp. 845-851.

Maidment, D., Miaou, S. and Crawford, M. (1985) 'Transfer function models of daily urban water use', Water Resources Research, Vol. 21 (4), pp. 425-432.

Makridakis, S. (1993) 'Accuracy measures: theoretical and practical concerns', International Journal of Forecasting, Vol. 9 (22), pp. 527-529.

Mohamed, A. S. and Savenije, H. H. G. (2000) 'Water Demand Management: Positive Incentives, Negative Incentives or Quota Regulation?', Physics and Chemistry of the Eart Part B - Hydrology Oceans and Atmosphere, Vol. 25(3), pp. 251-258.

Msiza, I., Nelwamondo, F. and Marwala, T. (2007) 'Artificial neural networks and support vector machines for water demand time series forecasting', IEEE International Conference on Systems, Man and Cybernetics, pp. 638-643.

Nasseri, M., Moeini, A., and Tabesh, M. (2011) 'Forecasting monthly urban water demand using Extended Kalman Filter and Genetic Algorithms', Expert Systems with Applications, Vol. 38, pp. 7387-7395. 
Pino, R., Parreño, J., Gómez, A. and Priore, P. (2008) 'Forecasting next-day price of electricity in the Spanish energy market using Artificial Neural Networks’, Engineering Applications of Artificial Intelligence, Vol. 21 (1), pp. 53-62.

Shvarster, L., Shamir, U. and Feldman, M. (1993) 'Forecasting Hourly Water Demand by Pattern Recognition Approach', Journal of Water Resources Planning and Management, Vol. 119 (6), pp. 611-627.

Willsie, R. H. and Pratt, L. H. (1974) 'Water use relationships and projection corresponding with regional growth Seattle region', Water Resources Bulletin, Vol. 10(2), pp. 360-371.

Winters, P. (1960) 'Forecasting sale by exponentially weighted moving averages', Management Science, Vol. 6, pp. 324-342. 


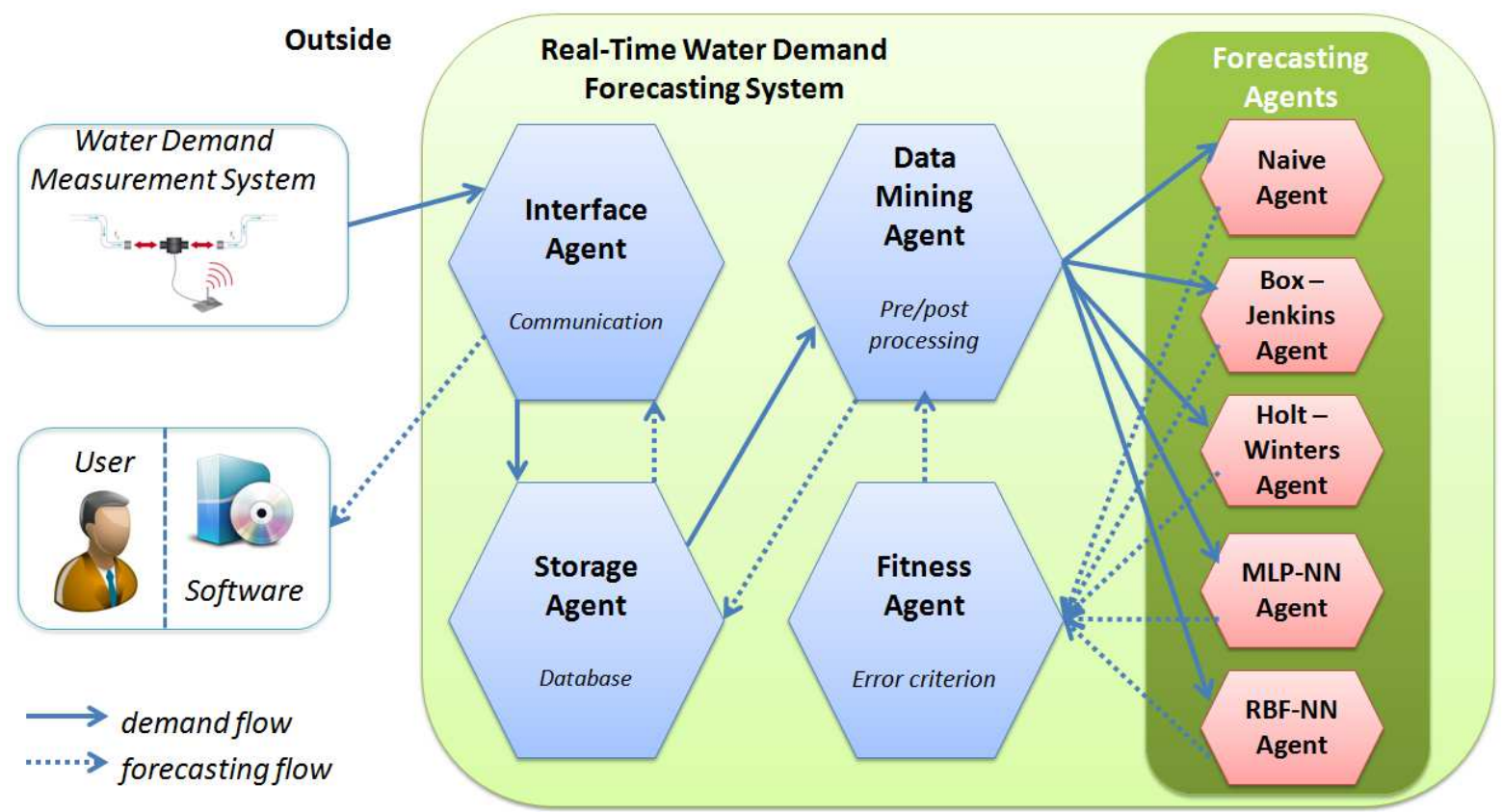

Fig. 1 General outline of the real-time water demand forecasting system, with the various agents that form it and the relationships among them (two main flows) and with the outside. 


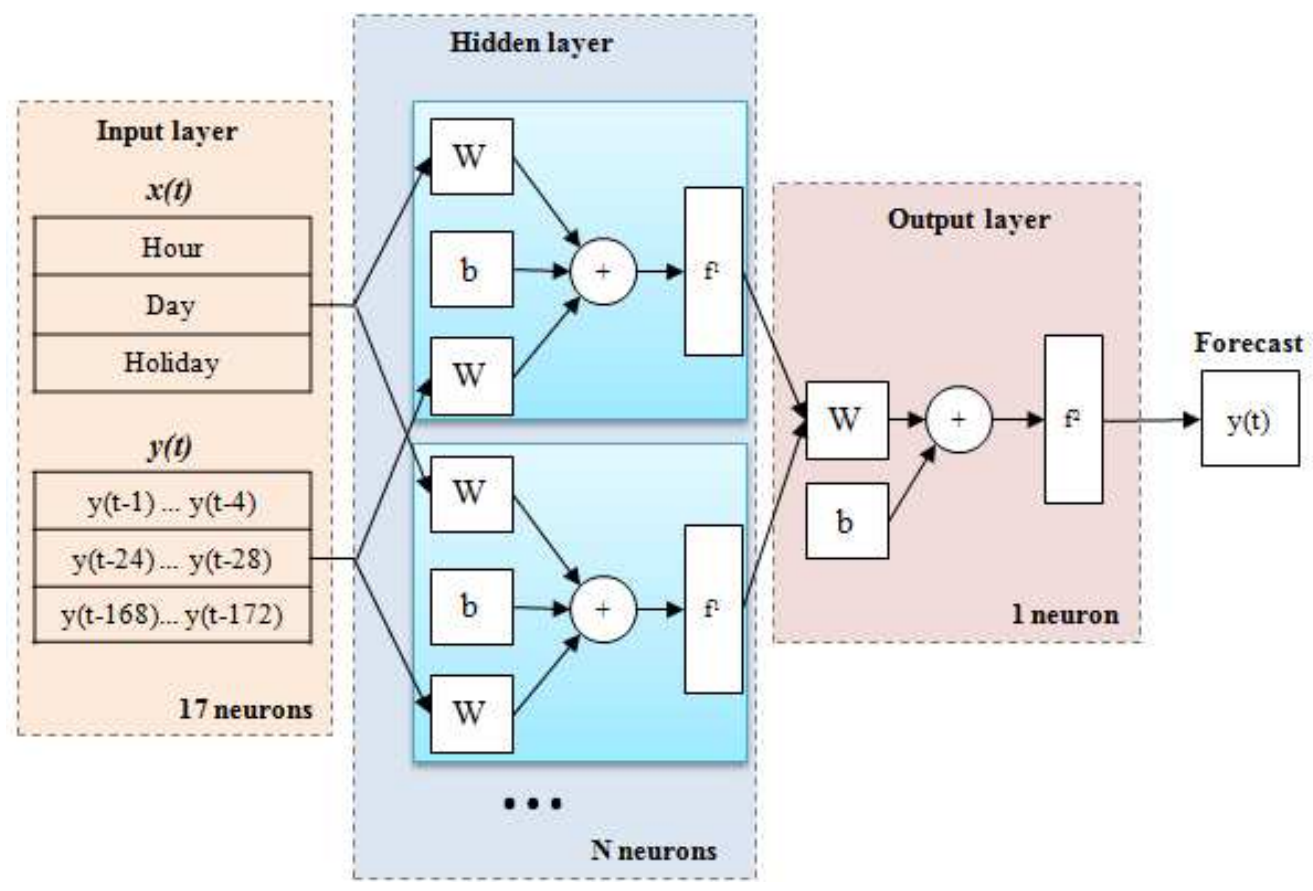

Fig. 2 General structure of the ANN with its three layers (input layer, hidden layer and output layer). The 17 predictor variables are: the day and hour of the demand to forecast, a binary variable that differentiates holidays and working days, and 14 past values related to the seasonality of the time series (from $y(t-1)$ to $y(t-4)$, from $y(t-24)$ to $y(t-28)$, from $y(t-168)$ to $y(t-172))$. The number of neurons in the hidden layer depends on the time series. The only output neuron is related to the variable to predict, so that it performs the forecast. 


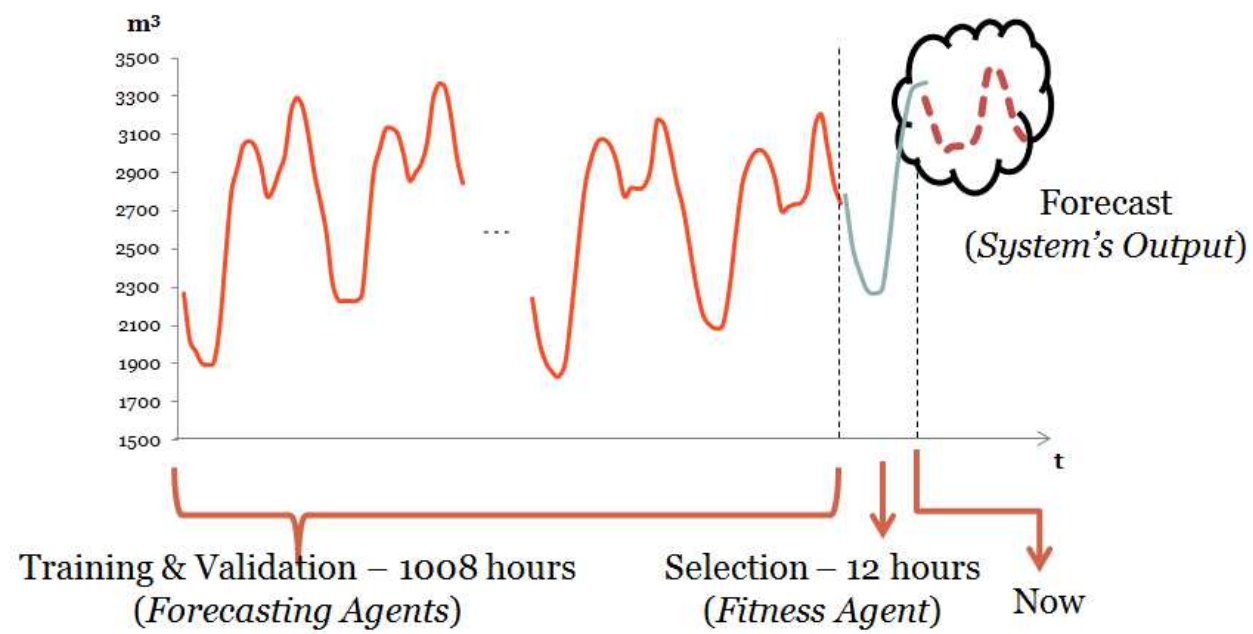

Fig. 3 The last 12 hours (both the demands and the forecasts of the five agents) are used to determine the best forecasting method in each moment, while the previous 1008 hours (only the demands) are used for the training and validation of the different forecasting methods, in order to choose the most appropriate model in each case (except the case of the Naïve Agent, whose functioning is much more simple). 

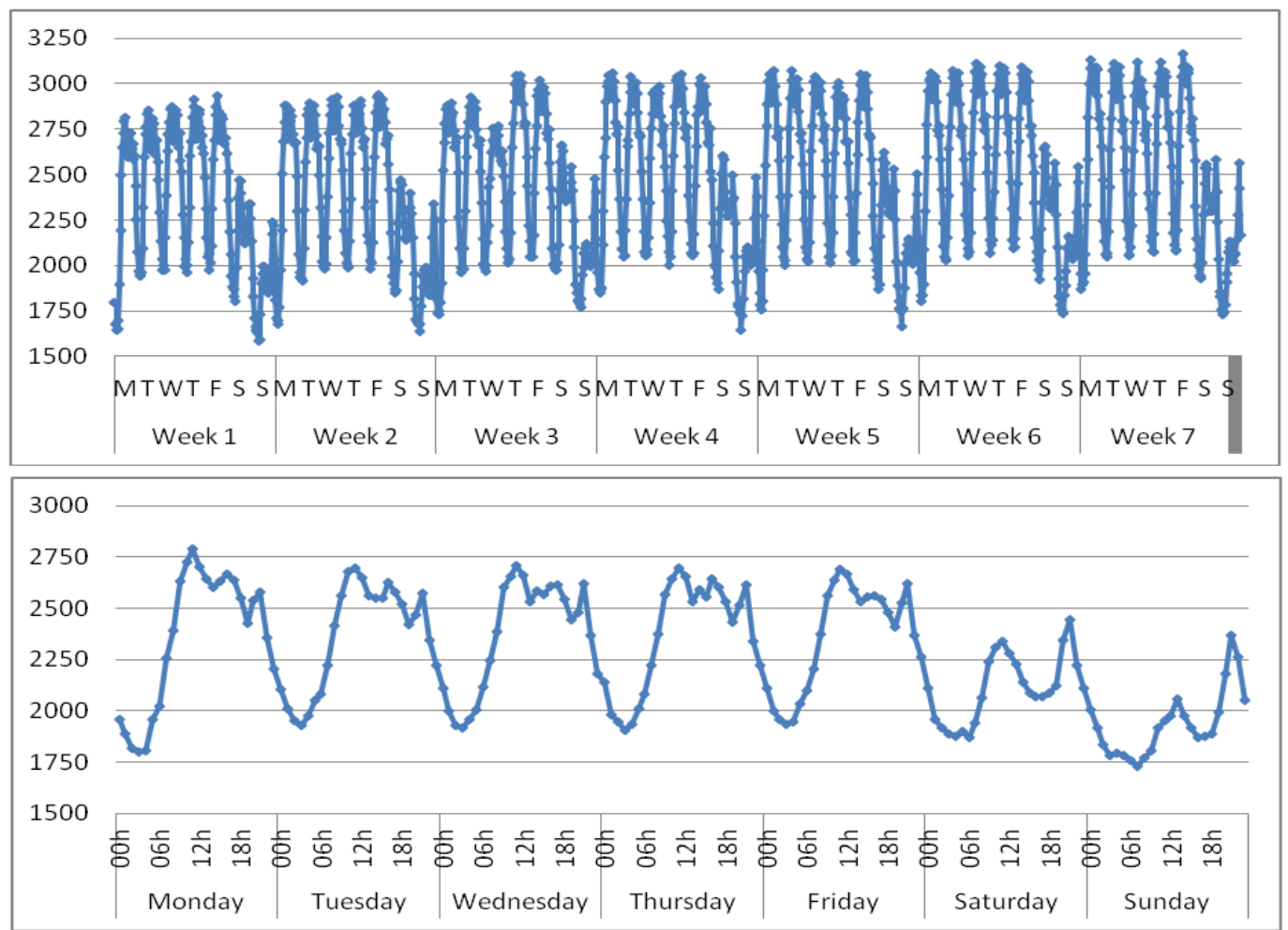

Fig. 4 Two extracts from the time series (values in cubic meters / hour). The top graph (time horizon: seven weeks) brings evidence of the weekly periodicity and its trend, and the graph below (time horizon: one week) shows the daily periodicity of the time series. 


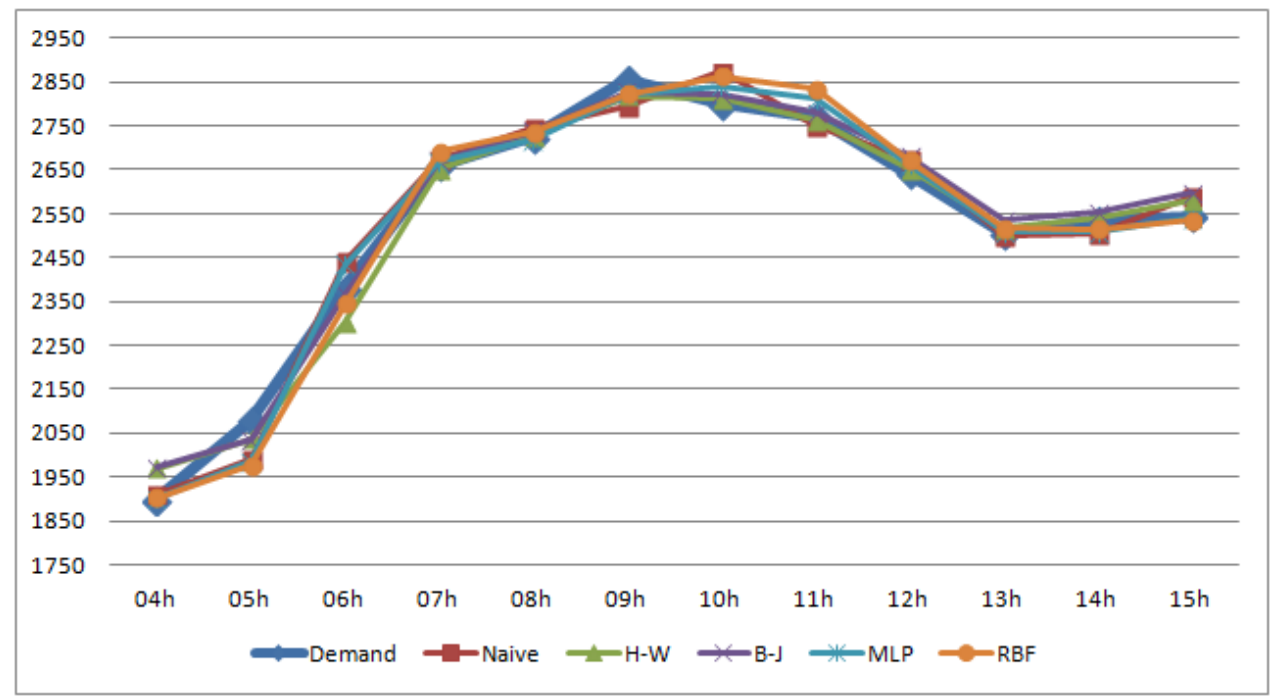

Fig. 5 Actual demand and various predictions for the forecasting period in test $I$ (values in cubic meters / hour). The MLP-NN forecasts is the one provided by the system (Forecasting MAPE 1,14\%), but the different among the various methodologies are much smaller than in the other cases studied. 


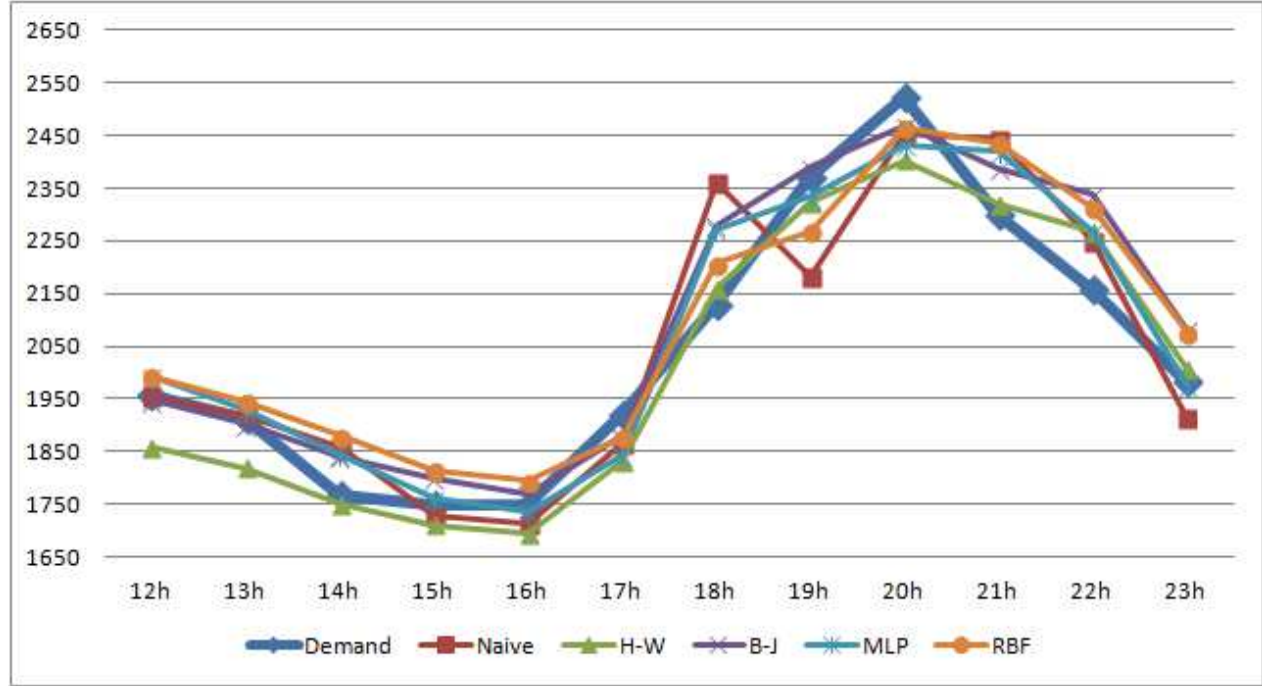

Fig. 6 Actual demand and various predictions for the forecasting period in test III (values in cubic meters / hour). The MLP-NN forecasts is the one provided by the system (Forecasting MAPE 2,95\%). 


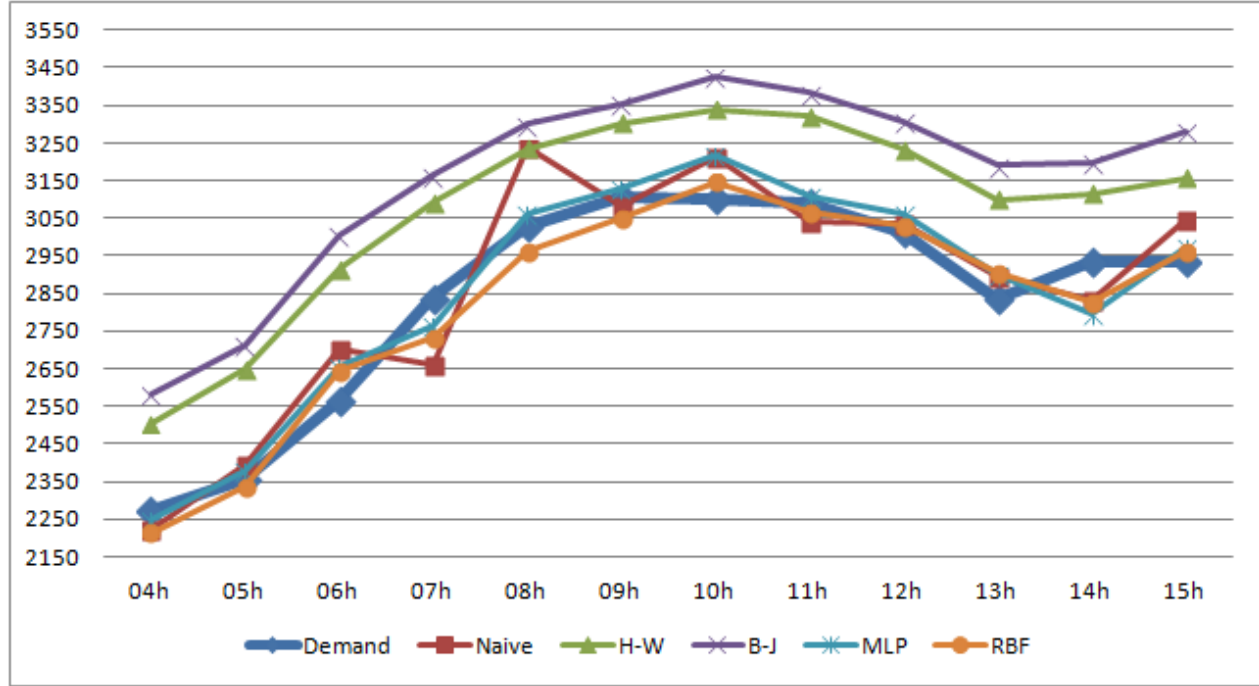

Fig. 7 Actual demand and various predictions for the forecasting period in test VI (values in cubic meters / hour). The statistical methods have big difficulties to forecast accurately. The MLP-NN forecast is the one provided by the system (Forecasting MAPE 2,98\%), although the RBF-NN forecast is slightly better. 
Table 1 Results of the numerical test. It contains the following five columns: (1) the beginning of the time period to predict (previous 1020 data are used by the system to forecast); (2) the forecasting method, by means of the Agent which performed the forecast; (3) its main feature chosen by the agent (that is to say, the HoltWinters model chosen, the ARIMA Model, and the structure of the ANN); (4) the MAPE calculated by the Fitness Agent (12 previous demands) and which determines its selection; and (5) the MAPE obtained in the prediction made by each agent. In order to calculate the Forecasting MAPE, we use the following 12 forecasts, with the aim of looking for consistency in our results.

\begin{tabular}{|c|c|c|c|c|}
\hline Forecasting Period & Forecasting Agent & Features & Fitness MAPE & Forecasting MAPE \\
\hline Test I & Naive & - & $1,94 \%$ & $1,60 \%$ \\
\hline Thursday & Holt-Winters & Simple seasonal & $2,56 \%$ & $1,17 \%$ \\
\hline May 14, 2009 & Box-Jenkins & $(0,1,6)(0,1,1)_{168}$ & $2,26 \%$ & $1,25 \%$ \\
\hline $04 \mathrm{~h}$ & MLP-NN & $17-8-1$ & $1,27 \%$ & $1,14 \%$ \\
\hline (working day) & RBF-NN & $17-10-1$ & $1,48 \%$ & $1,39 \%$ \\
\hline Test II & Naive & - & $1,74 \%$ & $1,98 \%$ \\
\hline Wednesday & Holt-Winters & Simple seasonal & $1,76 \%$ & $1,45 \%$ \\
\hline Sept. 8, 2010 & Box-Jenkins & $(0,1,3)(1,1,0)_{168}$ & $1,87 \%$ & $2,21 \%$ \\
\hline $16 \mathrm{~h}$ & MLP-NN & $17-9-1$ & $1,40 \%$ & $1,52 \%$ \\
\hline (working day) & RBF-NN & $17-10-1$ & $1,12 \%$ & $1,53 \%$ \\
\hline Test III & Naive & - & $3,59 \%$ & $3,93 \%$ \\
\hline Sunday & Holt-Winters & Additive & $4,34 \%$ & $3,03 \%$ \\
\hline June 7, 2009 & Box-Jenkins & $(0,1,3)(0,1,1)_{168}$ & $3,30 \%$ & $3,22 \%$ \\
\hline $12 \mathrm{~h}$ & MLP-NN & $17-6-1$ & $2,83 \%$ & $2,91 \%$ \\
\hline (weekend) & RBF-NN & $17-8-1$ & $4,29 \%$ & $3,85 \%$ \\
\hline Test IV & Naive & - & $3,51 \%$ & $2,50 \%$ \\
\hline Friday & Holt-Winters & Multiplicative & $3,69 \%$ & $2,62 \%$ \\
\hline Feb. 5, 2010 & Box-Jenkins & $(1,1,5)(0,1,1)_{168}$ & $2,63 \%$ & $8,04 \%$ \\
\hline $23 \mathrm{~h}$ & MLP-NN & $17-9-1$ & $2,39 \%$ & $2,48 \%$ \\
\hline (weekend) & RBF-NN & $17-11-1$ & $4,28 \%$ & $1,82 \%$ \\
\hline Test $V$ & Naive & - & $5,38 \%$ & $3,19 \%$ \\
\hline Tuesday & Holt-Winters & Simple Seasonal & $23,55 \%$ & $6,09 \%$ \\
\hline Dec. 8, 2009 & Box-Jenkins & $(1,1,1)(1,1,0)_{168}$ & $24,44 \%$ & $7,46 \%$ \\
\hline $18 \mathrm{~h}$ & MLP-NN & $17-4-1$ & $3,74 \%$ & $2,19 \%$ \\
\hline (holiday) & RBF-NN & $17-8-1$ & $6,20 \%$ & $1,78 \%$ \\
\hline Test VI & Naive & - & $4,37 \%$ & $3,22 \%$ \\
\hline Wednesday & Holt-Winters & Additive & $5,86 \%$ & $8,65 \%$ \\
\hline Oct. 13,2010 & Box-Jenkins & $(2,1,12)(0,1,1)_{168}$ & $8,04 \%$ & $11,48 \%$ \\
\hline $04 \mathrm{~h}$ & MLP-NN & $17-11-1$ & $2,98 \%$ & $2,03 \%$ \\
\hline (after holiday) & RBF-NN & $17-7-1$ & $3,79 \%$ & $2,00 \%$ \\
\hline
\end{tabular}

